\title{
Florence Nightingale (1820-1910), a 101 años de su fallecimiento
}

\author{
PABLO YOUNG ${ }^{1}$, VERÓNICA HORTIS DE SMITH ${ }^{\mathrm{a}}$, \\ MARÍA C. CHAMBI ${ }^{\mathrm{b}}$, BÁRBARA C. FINN ${ }^{1}$

\section{Florence Nightingale (1820-1910), 101 years after her death}

We herein describe Florence Nightingale's life and work. She is considered one of the pioneers in nursing practice. Her greatest success was during the Crimean war when, along with 38 voluntary nurses, she cleaned and refurbished the hospital in Scutari and reduced the mortality rate from 40 to $2 \%$. She used to make rounds at night in the wards under the light of a lamp, and therefore she was named "The Lady with the Lamp". Queen Victory gave her the Royal Red Cross and she was the first woman who was honored with the Order of Merit in 1907. She had solid knowledge on Statistics and Mathematics which were useful for her nursing job.

(Rev Med Chile 2011; 139: 807-813).

Key words: Crimean war; History of Nursing; Nursing, practical.

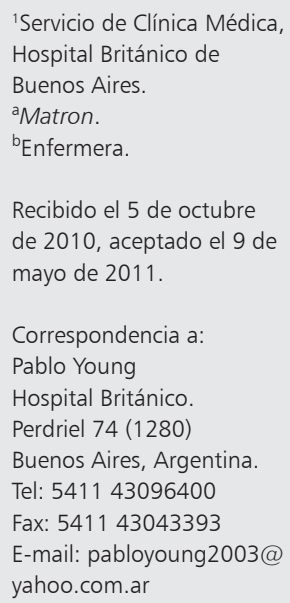

Correspondencia a: Pablo Young Hospital Británico. Perdriel 74 (1280) Buenos Aires, Argentina. Tel: 541143096400 Fax: 541143043393 E-mail: pabloyoung2003@ yahoo.com.ar

\section{Perspectiva histórica}

F lorence Nightingale (Figura 1) nació en Florencia, Italia, el 12 de mayo de 1820 y es considerada una de las pioneras en la práctica de la enfermería. Se le considera la madre de la enfermería moderna y verdadera creadora de una filosofía en la enfermería ${ }^{1-3}$.

La filosofía de la enfermería explica el significado de los fenómenos observados a través del análisis, el razonamiento y la argumentación lógica. Así pues, en esta categoría se han incluido los primeros trabajos que precedieron o condujeron a la construcción de los modelos teóricos y al desarrollo de los conocimientos en esta disciplina definiendo las bases de los futuros trabajos. En cambio los modelos conceptuales (estructuración de ideas y teorías) comprenden los trabajos de las llamadas grandes teorías o pioneras en el campo de la enfermería. O sea un modelo conceptual ofrece un marco de referencia para sus seguidores. La mayoría de los autores entienden que la obra de Nightingale está estrechamente relacionada con su orientación filosófica sobre la interacción paciente- entorno y los principios y reglas sobre los que sustentó su ejercicio profesional más que con el desarrollo de un modelo conceptual, aunque esto es todavía motivo de controversia ${ }^{4,5}$.

Ella definía la enfermedad como el camino que utiliza la naturaleza para desembarazarse de los efectos o condiciones que han interferido en la salud. Y definía salud diciendo que la salud es no solamente estar bien sino ser capaz de usar bien toda la energía que poseemos. La enfermería, entonces, es tanto ayudar al paciente que sufre una enfermedad a vivir, como poder o mantener el organismo del niño sano o del adulto en un estado tal que no padezca enfermedad ${ }^{6}$. Sostenía que para mantener una atención sanitaria adecuada era necesario disponer de un entorno saludable (aire puro, agua pura, alcantarillado eficaz, limpieza y luz) componentes que siguen teniendo vigencia al día de la fecha. Y son sustentados en el concepto de enfermería del Consejo Internacional de Enfermeras (CIE) cuando definen "la enfermería abarca los cuidados autónomos y en colaboración, que se presta a personas de todas las edades, grupos y comunidades, enfermos o sanos, en todos los contextos, e incluye la promoción de la salud, la prevención de la enfermedad, y los cuidados de 
los enfermos, discapacitados, y personas moribundas. Funciones esenciales de la enfermería son la defensa, el fomento de un entorno seguro, la investigación, la participación en la política de salud y en la gestión de los pacientes y los sistemas de salud, y la formación"6.

Se rebeló contra los prejuicios de su época y contra su destino de mujer, que debía permanecer en el hogar, y eligió la profesión de enfermera. Su mayor éxito fue su participación en la guerra de Crimea. Un informe suyo acerca de las condiciones de vida de los soldados heridos impulsó al secretario de Guerra Sidney Herbert a enviarla al campo de batalla. Ella y sus compañeras reformaron y limpiaron el hospital, e hicieron caer la tasa de mortalidad de $40 \%$ al $2 \%$.

Logró realizar su sueño de asistir a los enfermos después de enfrentarse a sus padres y familiares. $\mathrm{Su}$ madre Emily y su padre William se oponían a que su hija fuera enfermera, ya que estaba mal visto que una mujer perteneciente a una clase social alta desempeñara una tarea tan "denigrante"; la mujer debía casarse, formar y cuidar su familia. Sin embargo, Florence recibió ayuda de su abuelo materno, quien entendió su vocación por esta pro-

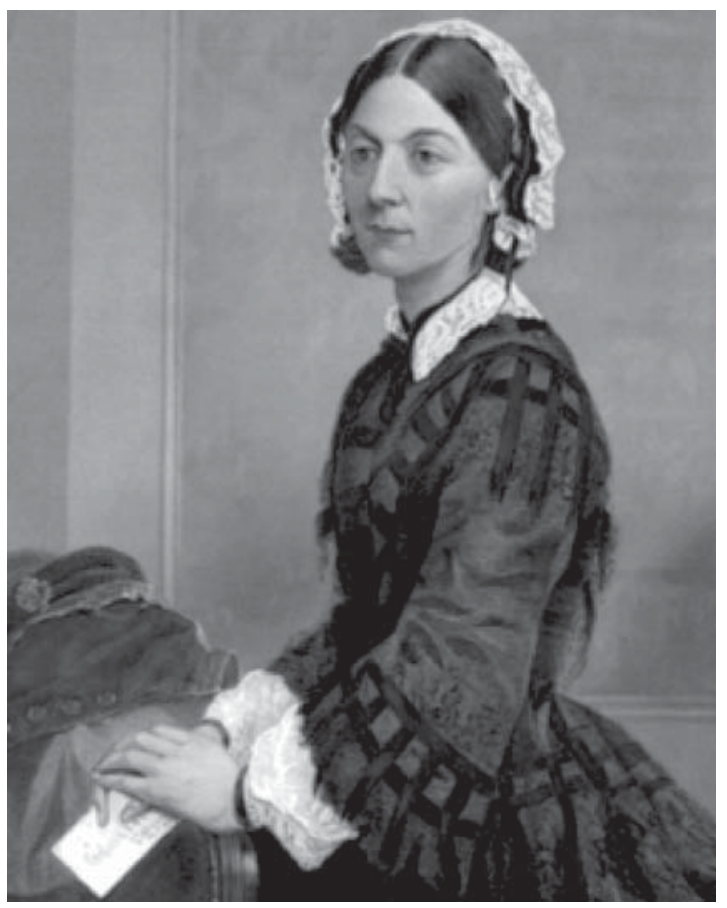

Figura 1. Florence Nightingale. fesión, y por otro lado ella misma buscó el apoyo de un amigo de la familia Nightingale, Samuel, médico de profesión. Nunca se casó; dedicó su vida al servicio del prójimo y de aquellos que más lo necesitaban, buscando la forma de mejorar su salud y en otros casos de hacer más llevaderos sus últimos días ${ }^{7}$.

Florence Nightingale es recordada sobre todo por su trabajo como enfermera durante la guerra de Crimea y por su contribución a la reforma de las condiciones sanitarias en los hospitales militares de campo.

\section{Su familia y estudios}

Nightingale lleva el nombre de la ciudad donde nació, Florencia. Sus padres, William Edward Nightingale y su esposa Frances Smith, viajaron por Europa durante los primeros dos años de su matrimonio. La hermana mayor de Florence había nacido un año antes en Nápoles.

William Nightingale se apellidaba en verdad Shore, cambia su apellido por Nightingale después de heredar a un pariente rico, Peter Nightingale de Lea, cerca de Matlock, Derbyshire. Las niñas crecieron en el campo y pasaban mucho tiempo en Lea Hurst en Derbyshire. Cuando Florence tenía unos cinco años su padre compró una casa llamada Embley cerca de Romsey en Hampshire. Con esto la familia pasaba los veranos en Derbyshire y el resto del año en Embley. Al viajar entre estos lugares visitaban Londres, y la Isla de Wight ${ }^{7,8}$.

En un principio, la educación de las niñas estuvo en manos de una institutriz, después su padre, educado en Cambridge, asumió esa responsabilidad. A Florence le encantaban sus lecciones y tenía una habilidad natural para estudiar. Bajo la influencia de su padre se familiarizó con los clásicos, Euclides, Aristóteles, la Biblia y temas políticos.

En 1840 suplicó a sus padres que la dejaran estudiar matemáticas en vez de trabajo de estambre y practicar las cuadrillas, pero su madre no aprobaba esta idea. Aunque William Nightingale amaba las matemáticas y había legado ese amor a su hija, la obligó a que siguiera estudiando temas más apropiados para una mujer. Después de muchas batallas emocionales, sus padres finalmente cedieron y comenzó su aprendizaje de matemáticas. Entre sus tutores estuvo Sylvester, quien desarrolló la teoría de invariantes junto con 
Cayley. Se dice que fue la alumna más destacada de Sylvester. Las lecciones incluían aritmética, geometría y álgebra. Una de las personas que también influyeron en ella fue el científico belga Quetelet. Él había aplicado métodos estadísticos a datos de varios campos, incluyendo las estadísticas morales o ciencias sociales?.

La religión jugó un papel importante en su vida. Aunque sus padres crecieron en la Iglesia Unitaria, Frances Nightingale prefirió una denominación más convencional y a las niñas las criaron en la fe anglicana.

La alianza entre Florence y Charles Dickens tuvo una indudable influencia como factor determinante en su definición de la enfermería y la atención sanitaria. Diálogos semejantes con otros intelectuales y reformadores sociales de aquellos días, como John Stuart Mill, Benjamin Jowett y Harriet Marineau, contribuyeron al desarrollo del pensamiento filosófico y lógico de Nightingale, que se trasluce de forma notoria en sus apreciaciones sobre la práctica de la enfermería.

\section{Florence Nightingale y la enfermería}

Nightingale desarrolló interés por los temas sociales de su época, pero en 1845 su familia se oponía firmemente a la idea de que ella trabajara en un hospital. Hasta ese entonces, el único trabajo de enfermería que había hecho había sido cuidar de parientes y amigos enfermos.

Mientras estaba de viaje por Europa y Egipto en 1849, tuvo la oportunidad de estudiar los distintos sistemas hospitalarios. A principios de 1850, inició su entrenamiento como enfermera en el Instituto de San Vicente de Paul en Alejandría, Egipto, que era un hospital perteneciente a la Iglesia Católica. Nightingale visitó el hospital del Pastor Theodor Fliedner en Kaiserwerth, cerca de Dusseldorf, Alemania en julio de 1850 y regresó a esa ciudad en 1851 para entrenarse como enfermera durante tres meses en el Instituto para Diaconisas Protestantes; y luego de Alemania se trasladó a un hospital en Saint Germain, cerca de París, dirigido por las Hermanas de la Caridad. A su regreso a Londres en 1853, tomó el puesto ad-honoren de Superintendente en el Establecimiento para damas en el número 1 de la calle Harley ${ }^{9,10}$.

Marzo de 1854 trajo consigo el inicio de la Guerra de Crimea que comenzó cuando Rusia invadió Turquía, este último en alianza con Inglaterra y Francia. La guerra finalizó en 1856. La mayor parte del conflicto tuvo lugar en la península de Crimea en el Mar Negro ${ }^{11}$. Aunque los rusos fueron derrotados en la batalla del río Alma, el 20 de septiembre de 1854, el periódico The Times criticó duramente las instalaciones médicas británicas. En respuesta a ello, Sidney Herbert, le pidió a Nightingale que se desempeñe como enfermera administradora para supervisar la introducción de enfermeras en los hospitales militares. Su título oficial era Superintendente del Sistema de Enfermeras de los Hospitales Generales Ingleses en Turquía. Nightingale llegó a Escutari, un suburbio asiático de Constantinopla (hoy Estambul) con 38 enfermeras, el 4 de noviembre de 1854. Firme e infatigable se ocupaba de su trabajo con tal criterio, sacrificio, valor, ternura y todo ello con una actitud tranquila y sin ostentación, que se ganaba los corazones de todos aquellos a quienes sus prejuicios de oficiales no les impedían apreciar la nobleza de su trabajo y de su carácter"12. En la organización de los servicios hospitalarios, en dos semanas logró montar una cocina para preparar la comida de 800 hombres; una lavandería en donde se desinfectaba la ropa de los pacientes, además dotó a los heridos y enfermos de 10 mil camisas compradas con los donativos que conseguía y de su propio dinero. "La dama de la lámpara", fue la denominación que le dieron a Florence los hospitalizados, debido a que por las noches recorría las salas con una lámpara (Figuras 2 y 3$)^{11,13}$.

Aunque ser mujer implicaba que tenía que luchar contra las autoridades militares, fue reformando el sistema hospitalario. Bajo condiciones indignas con soldados depositados sobre el suelo y con operaciones poco higiénicas, no sorprende que en Escutari enfermedades como el cólera y el tifus sucumbieran los hospitales. Esto implicaba que los soldados heridos tuvieran una probabilidad siete veces mayor de morir en el hospital que en el frente de batalla ${ }^{14,15}$. Mientras estuvo en Turquía, recolectó datos y organizó un sistema para llevar registro; esta información fue usada después como herramienta para mejorar los hospitales militares y de las ciudades. Sus conocimientos matemáticos se volvieron evidentes cuando usó los datos que había recolectado para calcular la tasa de mortalidad en el hospital. Estos cálculos demostraron que una mejora en los métodos sanitarios empleados, produciría una disminución 
en el número de muertes. Para febrero de 1855 la tasa de mortalidad había caído de $60 \%$ al 42,7\%. Mediante el establecimiento de una fuente de agua potable, así como usando su propio dinero para comprar fruta, vegetales y equipamiento hospitalario, para la primavera siguiente la tasa había decrecido otro $2,2 \%{ }^{15}$.

Nightingale usó esta información estadística para crear su Diagrama de Área Polar. Estos fueron usados para dar una representación gráfica de las cifras de mortalidad durante la Guerra de Crimea. Las muertes en los hospitales de campo británicos alcanzaron su máximo en enero de 1855 , cuando 2.761 soldados murieron por enfermedades contagiosas, 83 por heridas y 324 por otras causas, con un total de 3.168 muertes. El promedio de hombres en la armada ese mes fue de 32.393. Usando esta información, calculó una tasa de mortalidad de 1.174 por cada 10.000 , de los cuales 1.023 de cada 10.000 se debían a enfermedades infecciosas. De haber continuado así y sin la sustitución frecuente de tropas, entonces las enfermedades por sí mismas habrían acabado totalmente con el ejército británico en Crimea.

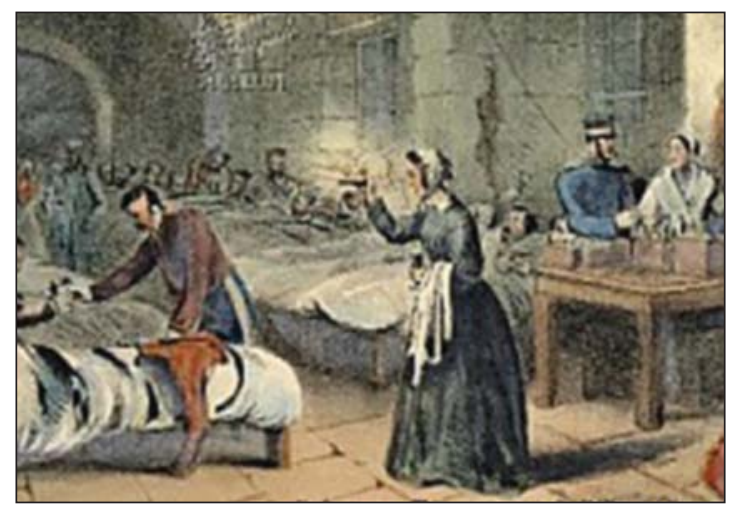

Figura 2. Florence Nightingale: "La Dama de la Lámpara".

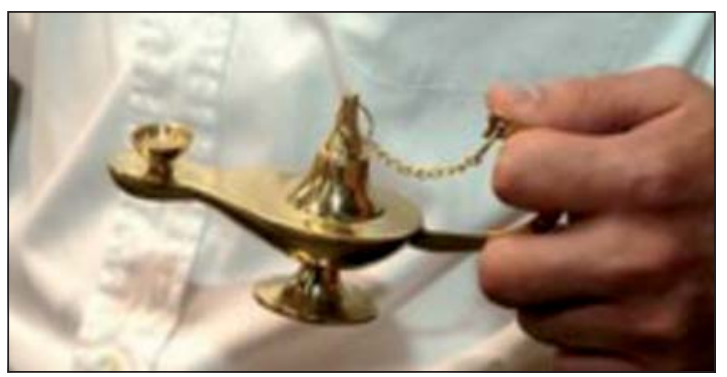

Figura 3. La Lámpara.
Sin embargo, estas condiciones insalubres no se limitaban a los hospitales militares. Al volver a Londres en agosto de 1856, cuatro meses después de la firma del tratado de paz, descubrió que en época de paz, los soldados de entre 20 y 35 años de edad tenían una tasa de mortalidad del doble de la de los civiles. Usando sus estadísticas, ilustró la necesidad de una reforma sanitaria en todos los hospitales militares. Al impulsar su causa, consiguió llamar la atención de la Reina Victoria y el Príncipe Alberto así como la del Primer Ministro, Lord Palmeston. Sus deseos de llevar a cabo investigación formal le fueron concedidos en mayo de 1857 y llevaron al establecimiento de la Comisión Real para la Salud del Ejército. Nightingale dejo de lado la atención pública y empezó a preocuparse por las tropas apostadas en la India ${ }^{16}$.

Fue pionera en la revolucionaria idea de que los fenómenos sociales pueden medirse y someterse al análisis matemático. Ella supo que cuando los valores individuales o profesionales entran en conflicto con los valores sociales, surge una posibilidad de inducir cambios en la sociedad y así lo hizo.

Aunque sus escritos se definen y analizan como una teoría, no contienen la complejidad y la verificabilidad propias de las modernas teorías de la enfermería. Así, en su enfoque no emanan investigaciones que pueden utilizarse para contrastar los aportes teóricos actuales. Por otra parte,

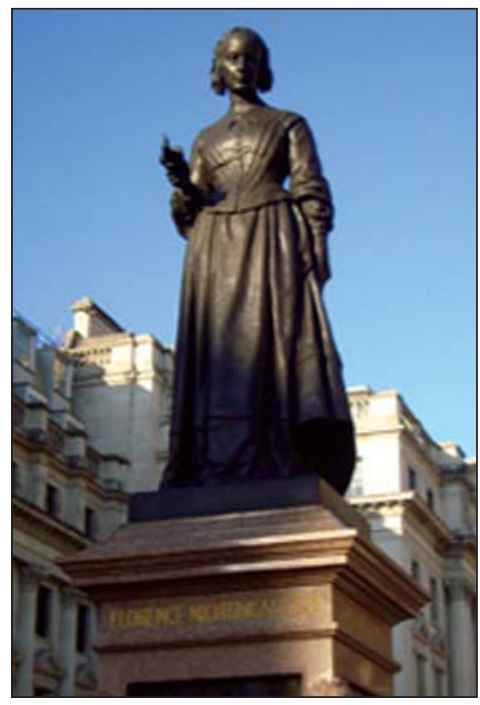

Figura 4. Monumento de Crimea, en Waterloo Place. 
los conceptos identificados por ella han servido como base de las teorías e investigaciones actuales, generando los modelos que se añaden a la ciencia y a la práctica moderna de la enfermería.

Este poema de Henry Wadsworth Longfellow (quien estuvo muy próximo a ella por su participación en la guerra) condensa la historia casi legendaria de Florence Nightingale y su obra maravillosa como enfermera durante la guerra de Crimea ${ }^{7}$.

Los heridos en la batalla, en lúgubres hospitales de dolor;

los tristes corredores, los fríos suelos de piedra.

¡Mirad! En aquella casa de aflicción Veo una dama con una lámpara. Pasa a través de las vacilantes tinieblas $y$ se desliza de sala en sala.

Y lentamente, como en un sueño de felicidad, el mudo paciente se vuelve a besar su sombra, cuando se proyecta en las obscuras paredes.

\section{Florence Nightingale y la Escuela de Enfermería}

En 1860 abrió la Escuela de Entrenamiento y Hogar Nightingale para Enfermeras en el hospital de St. Thomas en Londres, con 10 estudiantes ${ }^{6-10}$. Era financiada por medio del Fondo Nightingale, un fondo de contribuciones públicas establecido en la época en que estuvo en Crimea. La escuela se basaba en dos principios. El primero, que las enfermeras debían adquirir experiencia práctica en hospitales organizados especialmente con ese propósito. El otro era que las enfermeras debían vivir en un hogar adecuado para formar una vida moral y disciplinada. Con la fundación de esta escuela había logrado transformar la mala fama de la enfermería en el pasado en una carrera respetable. Nightingale respondió a la petición de la oficina de guerra británica y aconsejo sobre los cuidados médicos para el ejército en Canadá y también fue consultora del gobierno de los Estados Unidos sobre salud del ejército durante la Guerra Civil estadounidense.

Casi durante el resto de su vida estuvo postra- da en cama debido a una enfermedad contraída en Crimea (para algunos brucelosis, para otros fiebre tifoidea o fiebre de Crimea), que le impidió continuar con su trabajo como enfermera ${ }^{1}$. No obstante, la enfermedad no la detuvo de hacer campaña para mejorar los estándares de salud; publicó aproximadamente 150 libros. Uno de ellos se tituló "Notas sobre enfermería" (1860). Este fue el primer libro para uso específico en la enseñanza de la enfermería y fue traducido a muchos idiomas. Otras obras publicadas incluyen "Notas sobre los hospitales" (1859) y "Notas sobre la enfermería para las clases trabajadoras" (1861). En 1874 se convirtió en miembro honorífico de la American Statistical Association y en 1883 la Reina Victoria le otorgó la Cruz Roja Real por su labor. También fue la primera mujer en recibir la Orden al Mérito de mano de Eduardo VII en 19076-10.

Nightingale falleció en Londres, Inglaterra, el 13 de agosto de 1910 a los 90 años $^{17}$. Está enterrada en la Iglesia de St. Margaret, en East Wellow, cerca de Embley Park. El Monumento de Crimea, fue erigido en 1915 en Waterloo Place, Londres, para honrar la contribución que hizo Florence Nightingale a esa guerra y a la salud del ejército (Figura 4).

\section{Aportes a la enfermería}

Sus aportes dentro de la enfermería se consideran en dos niveles, en el ámbito general de la disciplina inició la búsqueda de un cuerpo de conocimiento propio, organizó la enseñanza y la educación de la profesión, inició la investigación en enfermería y fue la primera en escribir sobre la disciplina; y en el ámbito particular organizó la enfermería militar y fue la primera en utilizar la estadística, y el concepto de higiene dentro de la profesión ${ }^{18,19}$.

Además se considera esencial de la reforma Nightingale que la dirección de las escuelas debía estar en manos de una enfermera y no de un médico; había que seleccionar a las candidatas de acuerdo a sus aptitudes morales e intelectuales; y de impartir una enseñanza metódica en vez de ocasional, por medio de la práctica ${ }^{19,20}$.

$\mathrm{Su}$ aporte a la organización de los servicios de enfermería fueron su genio organizador y un ciento por ciento de eficacia. No habría sido nunca la dama de la lámpara, si no hubiera sido también la dama con un propósito y con capacidad ${ }^{11}$. 
Florence Nightingale marcó un hito en enfermería e inscribió para todas las generaciones de enfermeras el concepto de "cuidar de uno mismo, del entorno y al paciente", a través de toda su obra.

Teniendo en cuenta sus declaraciones sobre los deberes de las enfermeras en 1893, una comisión del Colegio Farrand del Hospital Harper de Detroit, redactó en reconocimiento a su trayectoria el Juramento de Florence Nightingale, el cual ha sido adoptado y adaptado, por la mayoría de Escuelas de Enfermería para tomar el juramento sus los egresados, dice así:

Juro ante Dios y teniendo por testigos a los aquí presentes, ayudar a las personas a desarrollar su capacidad de alcanzar una vida plena, ya sea promoviendo la salud o ayudando a restaurarla.

Juro brindar mis servicios situada en una visión integral del hombre, abarcando por igual su dignidad y su derecho al bienestar.

Juro no transgredir el derecho de mis pacientes, a su privacidad y confidencialidad, reconociendo que la intimidad del ser humano constituye uno de sus valores más preciados.

En el desempeño de mi profesión, me abstendré de todo tipo de discriminación, referente a ideología, religión o creencia, raza o nacionalidad, sexo, enfermedad o minusvalía de las personas.

Juro poner todo mi esfuerzo y conocimiento en brindar cuidados de la más alta calidad, en las distintas etapas de la vida de mis pacientes, hasta en sus últimos días.

Si leemos sus cuadernos de notas, encontraremos el secreto de aquel celo con que consagró su vida a los demás, porque su extraordinaria inteligencia se alimentó en el constante estudio del misticismo cristiano, y su vida fue, manifiestamente, la expresión de sus creencias religiosas. He aquí una nota, que la representa típicamente: "El camino para vivir con Dios es vivir con las ideas, no meramente pensar sobre los ideales, sino actuar y sufrir por ellos. Los que tienen que trabajar como hombres y mujeres deben sobre todas las cosas tener un ideal espiritual, que es su finalidad, siempre presente. El estado místico es la esencia del sentido común"16,21,22.

A Florence Nightingale se la considera por todo lo antedicho la precursora de la enfermería moderna, y la fecha de su nacimiento se ha designado como el día internacional de la enfermería ${ }^{22}$.

\section{La Escuela de Enfermería en Argentina y en nuestro hospital}

En 1904 se realizó el Segundo Congreso Latinoamericano de Medicina, y la Dra. Cecilia Grierson (1859-1934) (primera médica Argentina) aprovechó para dirigirse a sus colegas del continente, "lo que nosotros, los médicos latinoamericanos estamos discutiendo y poniendo sobre votación en los Congresos, está resuelto y puesto en práctica en Europa. No hay hospital sin escuela de enfermería". En 1886, fundó la Escuela de Enfermeras del Círculo Médico Argentino, primera Escuela de Enfermería de Argentina, la que dirigió hasta 1913 y que desde 1934 lleva su nombre ${ }^{23}$.

El Hospital Británico de Buenos Aires es fundado por el Reverendo Barton Lodge en el año $1844^{24}$. La Escuela de Enfermería del Hospital Británico de Buenos Aires (segunda del país) comenzó a funcionar en el año 1890, bajo el Sistema Nightingale, con una formación sistemática de tres años, capacitando a mujeres allegadas a la colectividad inglesa, para ejercer con mayores conocimientos una tarea singular. En sus comienzos funcionó como un servicio a la comunidad, las clases se daban en inglés, y la mayoría de los pacientes también tenían esa misma lengua ${ }^{18}$. En 1882 se contrató a la Srta. E. Taylor quien viajó desde Inglaterra para ocupar el cargo de Jefa de Enfermeras dentro del Hospital y en el año 1889 llegaron cuatro enfermeras egresadas del St. Thomas Hospital de Londres alumnas directas de Florence Nightinga$\mathrm{le}^{18,20,24}$. Una de ellas fue la Sta. Ana Eammes (del resto no existen registros de sus nombres) quien en 1905 es nombrada Matron, y a la vez fue la primera que ocupó el cargo como Directora de Enfermería porque sólo se dedicó a la supervisión de sus enfermeras, dado que las anteriores tenían una actuación más relacionada con lo doméstico. En 1908 se adoptó la malla curricular formativa de la Escuela del Hospital Santo Tomás de Londres 
con un plan de estudios y prácticas. En el año 1964 es reconocida oficialmente por el Ministerio de Salud Pública de la Nación. En 1994, se asocia a la Escuela de Enfermería de la Facultad de Medicina de la Universidad de Buenos Aires, incorporándose como Unidad Docente de Enfermería.

Desde su creación y de manera ininterrumpida todos los años asistimos a la graduación de las enfermeras mejor formadas de nuestro país, continuando con la mística de Florence Nightingale.

En Argentina la formación en enfermería se desarrolla en el nivel superior de enseñanza en establecimientos universitarios y terciarios no universitarios. Existen 43 escuelas de enfermería universitarias, de las cuales 30 corresponden a universidades nacionales públicas (como la de nuestro hospital) y 13 a universidades o institutos universitarios privados ${ }^{25}$.

Vaya este trabajo con dedicación al rol de la Enfermería dentro de la actividad del hospital por cuanto es el que permanece al lado del paciente día y noche para atender sus requerimientos y asistirlo durante la recuperación de su salud. De su respuesta eficaz depende la mayoría de las veces el pronóstico y la efectividad del tratamiento.

Agradecemos a la Licenciada Zulma Silva, actual Directora de la Escuela de Enfermería por la lectura crítica del manuscrito.

\section{Referencias}

1. Young DAB. Florence Nightingale’s fever. Br Med J 1995; 311: 1711-4.

2. Raile Alligood M, Chog Choi E. Evolución del desarrollo de las teorías de enfermería. En: Marrimer Tomey A, Raile Alligood M, Editores. Modelos y teorías en enfermería. Madrid, España: Editorial Harcourt Brace; $4^{\circ}$ Ed; 1999. p. 3-15.

3. Pfettscher SA, de Graaf KR, Marriner Tomey A, Mossman CL, Slebodnik M. Florence Nightingale. La enfermería moderna. En: Marrimer Tomey A, Raile Alligood M, Editores. Modelos y teorías en enfermería. Madrid, España: Editorial Harcourt Brace; 4º Ed; 1999. p. 69-85.

4. Fawcett J. Analysis and evaluation of contemporary nursing knowledge: models and theories. Philadelphia: Editorial Davis Company; 2000. p. 9-24.

5. Monty EJ, Tingen MS. Multiple paradigms of nursing science. Adv Nurs Sci 1999; 21: 64-80.
6. Monteiro LA. Florence Nightingale on Public Health Nursing. Am J Public Health 1985; 75: 181-6.

7. Tan SY, Holland P. Florence Nightingale (1820-1910): founder of modern nursing. Singapore Med J 2006; 47: 185-6.

8. Dossey BM. Florence Nightingale: a 19th-century mystic. J Holist Nurs 2010; 28: 10-35.

9. Miracle VA. The life and impact of Florence Nightingale. Dimens Crit Care Nurs 2008; 27: 21-3.

10. Stanley D, Sherratt A. Lamp light on leadership: clinical leadership and Florence Nightingale. J Nurs Manag 2010; 18: 115-21.

11. Stanley D. Lights in the shadows: Florence Nightingale and others who made their mark. Contemp Nurse 2007; 24: 45-51.

12. Jackson B. Florence Nightingale and the Crimean War. Trans Med Soc Lond 2002; 118: 31-6.

13. Olshansky E. In celebration of Florence Nightingale: 2010 as the year of the nurse. J Prof Nurs 2010; 26: 197 8.

14. Fee E, Garofalo ME. Florence Nightingale and the Crimean War. Am J Public Health 2010; 100: 1591.

15. Dossey BM. Florence Nightingale: her Crimean fever and chronic illness. J Holist Nurs 2010; 28: 38-53.

16. Ellis $\mathrm{H}$. Florence Nightingale: nurse and public health pioneer. Br J Hosp Med (Lond) 2010; 71: 51.

17. Florence Nightingale. California State Journal of Medicine 1910; 8: 289-90.

18. Salamendi de Cattaneo V. Escuela de Enfermería del Hospital Británico de Buenos Aires. Revista Temas de Enfermería 1994; 10: 27-35.

19. Attewell A. Florence Nightingale (1820 - 1910). Perspectivas 1998; 1: 173-189.

20. Molina TM. Historia de la Enfermería. Editorial Interamericana. Buenos Aires, 1973.

21. Nightingale F. Notas sobre enfermería: Qué es y qué no es. Barcelona, España: Editorial Salvat; 1990. p. 1-138.

22. Burgos Moreno M, Paravic Klijn T. Enfermería como profesión. Revista Index de Enfermería 2007; 16: 65-69. Disponible en: www.scielo.cl [Consultado el 11 de abril de 2011].

23. Zuckerberg C. Cecilia Grierson (1859-1934). Medicina (B Aires) 2005; 65: 557-8.

24. Warneford-Thomson HF. The British Hospital of Buenos Aires. A History 1844-2000. Colin Sharp editors. 2001. p. 3-177.

25. González A, Castro C, Moreira S, Cerino S, Correa Rojas MDV, Atzemian R, et al. Situación de la formación en las escuelas de enfermería terciarias no universitarias de la República Argentina, 2007. Rev Argent Salud Pública 2010; $1: 28-32$. 